\title{
Appropriate conversion from valproate monotherapy to lamotrigine monotherapy in Japanese women with epilepsy
}

Takamichi Yamamoto ${ }^{1}$, Yuichi Kubota ${ }^{2}$, Hirohiko Murayama ${ }^{3}$, Hirofumi Ozeki ${ }^{3}$, Yotaro Numachi ${ }^{3}$, Akio Ikeda ${ }^{4}$, on behalf of the Lamictal 200776 Study Group

\author{
${ }^{1}$ Seirei Hamamatsu General Hospital, Shizuoka, Japan \\ ${ }^{2}$ Department of Neurosurgery, Asakadai Central General Hospital, Saitama, Japan \\ ${ }^{3}$ Development and Medical Affairs, GlaxoSmithKline K.K., Tokyo, Japan \\ ${ }^{4}$ Department of Epilepsy, Movement Disorders and Physiology, Graduate School of Medicine, Kyoto \\ University, Kyoto, Japan
}

Key words: Epilepsy, valproate, lamotrigine, conversion algorithm, childbearing potential, Japanese women, skin rash

Received: August 26, 2016; Accepted: November 9, 2016

\begin{abstract}
Purpose: Exposure to sodium valproate (VPA) during pregnancy may increase the risk of fetal malformations and cognitive developmental deficits. We therefore assessed a clinically practical method to change treatment from VPA monotherapy to lamotrigine (LTG) monotherapy in Japanese women of childbearing potential whose seizures were controlled by VPA.

Methods: In an open-label, single arm, multicenter study, we evaluated the reduction in VPA dose and change in seizure frequency from baseline when VPA monotherapy was switched to LTG monotherapy in female patients by the following protocol. (1) The LTG dose was increased up to 200 $\mathrm{mg}$ /day while the initial VPA dose was maintained. (2) The VPA dose was reduced to $0 \mathrm{mg} /$ day while the LTG dose was maintained. (3) When VPA was removed, the LTG dose was simultaneously increased by up to $100 \mathrm{mg} /$ day. (4) The LTG dose was further adjusted. Due to the risk of break-through seizures during adjustment of VPA and LTG doses, LTG trough serum concentration was measured for seizure control.

Results: Of 33 patients enrolled, 20 completed the entire protocol, with VPA removal in 19 patients. Thirteen patients were withdrawn from the study during LTG escalation, mainly due to LTG-related skin rash $(\mathrm{n}=8)$ in the early stage of LTG initiation (10 days on average). Seizures were observed once in 2 patients during LTG monotherapy ( 7 and 13 weeks after VPA removal).

Discussions: By paying careful attention to the occurrence of skin rash or any sign of hypersensitivity at the beginning of LTG escalation, Japanese women receiving VPA monotherapy can be converted to LTG monotherapy.
\end{abstract}




\section{Introduction}

Sodium valproate (VPA) is widely used in the treatment of epilepsy, bipolar disorder and migraine in Japan and other countries, although various new antiepileptic drugs (AEDs) such as lamotrigine (LTG) are now available for both monotherapy and add-on therapy. The Japanese guideline for the treatment of epilepsy also recommends VPA as the first-line treatment for generalized seizures and as the second-line treatment for partial seizures [1]. However, several studies have shown that prenatal exposure to VPA may cause fetal malformations and cognitive developmental deficits in a dose-dependent manner, whereas such risk is relatively low for newer AEDs [2, 3]. The US Food and Drug Administration (FDA) therefore announced that VPA should not be used for preventing migraine in pregnant women, and that VPA should be restricted for the treatment of epilepsy and bipolar disorder and used only if other drugs are not effective or appropriate [4]. The FDA also announced that VPA use should not be allowed in women of childbearing potential in any circumstance, unless VPA is essential to the management of the women's medical conditions [4]. Similarly, the Japanese guideline recommends not using VPA as AED for women of childbearing potential [1].

Several open-label studies have evaluated the conversion from VPA monotherapy to LTG monotherapy in patients with poorly controlled partial seizures or generalized seizures [5, 6] and juvenile myoclonic seizures $[7,8]$ using VPA monotherapy. In a study by Sale et al. [6], the LTG dose was increased gradually up to $200 \mathrm{mg}$ /day while previously stable VPA doses were maintained during the initial 8 weeks. Subsequently, the VPA dose was decreased gradually to $0 \mathrm{mg}$ /day over the next 2-6 weeks, and the LTG dose was increased to $300 \mathrm{mg} /$ day at a VPA dose of 250 $\mathrm{mg}$ /day and further increased to $400 \mathrm{mg}$ /day when VPA was removed. Finally, the LTG dose was increased up to the maintenance dose of $500 \mathrm{mg} /$ day. Using this protocol, LTG monotherapy was achieved in 48 of 77 patients, and seizure frequency was increased in only 2 patients due to noncompliance. These results are consistent with those of other studies [5, 7, 8]. Buchanan [7] also showed that some adverse events associated with VPA, such as weight gain, were minimized by converting to LTG monotherapy.

In the present study, we modified the clinical methods of treatment changes described previously according to the dosing regimen of LTG approved in Japan and the clinical practice in Japan. Considering the potential benefits of removing VPA in women of childbearing potential and the undesirable risk of increasing seizure frequency in an uncontrollable manner, we enrolled Japanese pre-menopausal women (aged $\geq 15$ years) whose seizures were well controlled by VPA monotherapy (seizure-free), and explored whether the VPA dose can be reduced by introducing LTG without altering seizure frequency.

\section{Methods}

This phase IV, open-label, single-arm, multicenter study (ClinicalTrials.gov identifier: NCT02100644) was conducted between April 2014 and May 2015 by 28 investigators at 7 sites, in accordance with the Good Clini- 
cal Practice (GCP) guidelines, ethical principles of the Declaration of Helsinki, and subject privacy requirements and all other relevant regulations in Japan. The protocol and amendments were approved by the institutional review boards (IRB), and written informed consent was obtained from all patients (for patients younger than 20 years of age: parent's consent in addition to patient's assent).

\section{Patients}

The target number of patients was set at 30 since the purpose of this study was to describe the experience of converting VPA monotherapy to LTG monotherapy, and not to evaluate this conversion algorithm statistically. Patients who met the following criteria were enrolled: (i) partial seizures (including secondary generalized seizures) or tonicclonic seizures (with myoclonus but without any other generalized seizure types); (ii) seizure-free and on stable doses of VPA alone (400-1200 mg/day) for at least 12 weeks prior to study entry (VPA monotherapy); (iii) Japanese pre-menopausal non-pregnant women aged $\geq 15$ years; and (iv) no history of hypersensitivity to LTG or rash caused by AEDs including LTG.

\section{Study design and procedures}

As shown in Figure 1, the study consisted of five consecutive phases: a retrospective screening phase for 12 weeks; LTG escalation phase for the initial 8-18 weeks; VPA reduction phase for the subsequent 4-16 weeks; LTG monotherapy phase for 12 weeks (5 visits); and follow-up phase for the last 1-4 weeks (1 visit). The durations (numbers of visits) of the LTG escalation phase and the VPA reduction phase were determined by the investigators based on the patients' seizure condition and safety. As an example, the entire period of study was 50 weeks for the slowest titration.

Before obtaining written informed consent, the medical record of each patient was retrospectively reviewed to confirm seizure status and type, the stable maintenance dose of VPA, and concomitant medications in order to confirm that the enrolment criteria outlined above were satisfied. Once eligibility was determined and informed consent was obtained, LTG was administered according to the dosing regimen approved in Japan.

The LTG dose was started from $25 \mathrm{mg} /$ day

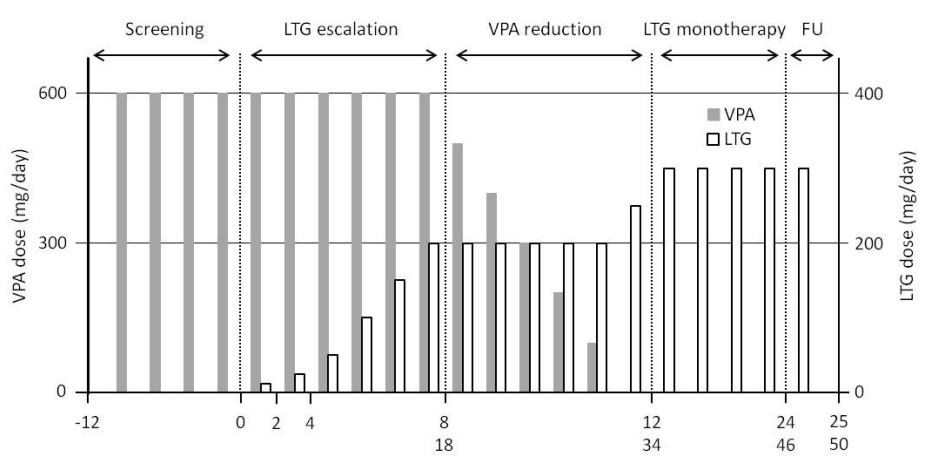

Figure 1. Study design

Schematic illustration of the VPA monotherapy to LTG monotherapy conversion algorithm. The VPA and LTG dosing steps are shown in gray and white, respectively. The durations (the numbers of visits) of the LTG escalation phase and VPA reduction phase were determined by the investigators. The horizontal axis shows the fastest (upper) and the slowest (lower) titration schedule. VPA: sodium valproate, LTG: lamotrigine, FU: follow up. See details in the text.

every other day for the first 2 weeks, and then increased to $25 \mathrm{mg}$ /day for the next 2 weeks. Thereafter, the daily LTG dose was increased gradually by 25-50 mg every 1-2 weeks up to $200 \mathrm{mg} /$ day (once or twice daily). If skin rash occurred during this phase and thereafter, the patients were immediately evaluated by the 
investigators and dermatologists, and were withdrawn from the study unless the rash was clearly not related to LTG. If other adverse events such as dizziness or sleepiness were observed, the LTG dose was decreased to 100 $\mathrm{mg}$ /day. In all other cases, the target LTG dose of $200 \mathrm{mg}$ /day should be reached to reduce the risk of seizures.

During the VPA reduction phase, the VPA dose was reduced gradually while the LTG dose (twice daily) was maintained. The quantity and interval of VPA dose reduction were determined by the investigators until the VPA dose reached $300 \mathrm{mg} / \mathrm{day}$. From 300 to $0 \mathrm{mg} /$ day, the daily dose was decreased by $100 \mathrm{mg}$ at an interval of a week or longer. When VPA was removed, it was necessary to increase the daily LTG dose by $25-100 \mathrm{mg} /$ day to reduce the risk of seizures due to loss of VPAinduced inhibition of hepatic enzymes associated with LTG metabolism resulting in reduction of serum half-life of LTG $[6,9]$. During this phase and thereafter, if there was any seizure concern or if a seizure had actually occurred, VPA dose increase was allowed when VPA was used concomitantly, and either LTG dose increase or addition of VPA and/or another AED was allowed after VPA was removed.

During the LTG monotherapy phase, the daily LTG dose should be increased by steps of $25-100 \mathrm{mg}$ every 1-2 weeks to a target dose of $300 \mathrm{mg} /$ day and was maintained at this dose. However, if there was any efficacy and/or safety concern, the LTG dose was adjusted to $100-400 \mathrm{mg} /$ day. Finally, at the follow-up phase, change in treatment was allowed, according to the investigators' judgement of the appropriate treatment for individual patients.

\section{Efficacy and safety outcome measures}

The primary endpoints were the percentage of patients in whom the VPA dose could be reduced, and the percentage of VPA dose reduction from baseline (week 0) at the last visit of the LTG monotherapy phase. The secondary endpoints were the changes in seizure frequency recorded in a seizure diary, completion and discontinuation rates, and changes in patients' quality of life (QOL) assessed using a questionnaire (QOLIE-31-P [10] for ages $\geq 18$ years, QOLIE-AD-48 [11] otherwise). The safety and tolerability were evaluated by assessment of all adverse events reported in addition to the results of standard laboratory tests (biochemistry, hematology, and urinalysis), vital signs, and electrocardiography (ECG).

\section{Pharmacokinetics}

The LTG trough serum concentration was measured at each visit during the VPA reduction phase and LTG maintenance phase, allowing the investigators to determine the quantity and interval of VPA dose reduction (as well as subsequent adjustment of LTG dose) and to control seizures throughout the study period. Therefore, blood samples were collected after a sufficient interval from the last LTG dosing (not specified in the protocol, but generally 8-16 hours). If blood samples were collected multiple times at the same VPA dose, the last sample was used for analysis.

\section{Data analyses}

Data were summarized with descriptive statistics including standard deviation (SD) and $95 \%$ confidence interval (CI). For some 
measures, post-hoc analyses were performed (see Results section for details). The KaplanMeier method was also used to calculate the time of withdrawal from study due to LTGrelated skin rash as adverse event and other reasons, or study completion as censored.

\section{Results}

\section{Patients}

The demographic and disease data are summarized in Table 1. In total, 33 seizurefree patients were enrolled in the study. The most commonly reported seizure types at baseline were primarily generalized tonicclonic seizures $(n=23)$. The initial VPA dose at baseline was $571.2 \pm 168.18 \mathrm{mg}$ /day (mean $\pm \mathrm{SD}$ ) ranging from $400-1100 \mathrm{mg} /$ day. The age of the patients when informed consent was obtained was $25.9 \pm 7.69$ (range: 15-45) years.

As shown in Figure 2, all 33 patients received LTG treatment and 20 completed the entire study period. Thirteen patients discontinued the study mainly due to adverse events including skin rash $(\mathrm{n}=8)$ and sub-acute necrotizing lymphadenitis $(n=1)$. The remaining 4 patients discontinued due to consent withdrawal $(\mathrm{n}=3)$ and protocol deviation of a history of skin rash during carbamazepine treatment $(\mathrm{n}=1)$.

\section{Efficacy analyses}

Of 20 patients who completed the entire study period, 19 patients achieved LTG monotherapy. In these 19 patients, an LTG dose of $200 \mathrm{mg} /$ day was reached at 10-17 weeks (median: 16 weeks) during the LTG escalation phase, except 1 patient whose LTG dose was decreased to $100 \mathrm{mg} /$ day due to LTG-
Table 1. Demographic data and disease characteristics

\begin{tabular}{|c|c|}
\hline & $\mathrm{n}(\%)$ \\
\hline $\begin{array}{l}\text { Gender } \\
\text { Female } \\
\text { Male }\end{array}$ & $\begin{array}{c}33(100 \%) \\
0(0 \%)\end{array}$ \\
\hline $\begin{array}{l}\text { Age (years) } \\
\quad<15 \\
\geq 15 \text { and }<20 \\
\geq 20 \text { and }<30 \\
\geq 30 \text { and }<40 \\
\geq 40 \text { and }<50 \\
\geq 50\end{array}$ & $\begin{array}{c}0(0 \%) \\
7(21.2 \%) \\
16(48.5 \%) \\
8(24.2 \%) \\
2(6.1 \%) \\
0(0 \%)\end{array}$ \\
\hline $\begin{array}{l}\text { Initial VPA dose }(\mathrm{mg} / \text { day }) \\
\quad<400 \\
\geq 400 \text { and }<500 \\
\geq 500 \text { and }<600 \\
\geq 600 \text { and }<700 \\
\geq 700 \text { and }<800 \\
\geq 800 \text { and }<900 \\
\geq 900 \text { and }<1000 \\
\geq 1000 \text { and }<1100 \\
\geq 1100 \text { and }<1200 \\
\geq 1200\end{array}$ & $\begin{array}{c}0(0 \%) \\
12(36.4 \%) \\
1(3.0 \%) \\
13(39.4 \%) \\
2(6.1 \%) \\
4(12.1 \%) \\
0(0 \%) \\
0(0 \%) \\
1(3.0 \%) \\
0(0 \%)\end{array}$ \\
\hline Seizure types at baseline & \\
\hline $\begin{array}{l}\text { Partial seizures } \\
\text { Simple partial seizures } \\
\text { Complex partial seizure } \\
\text { Secondarily generalized seizures }\end{array}$ & $\begin{array}{l}4(12.1 \%) \\
9(27.3 \%) \\
8(24.2 \%)\end{array}$ \\
\hline $\begin{array}{l}\text { Generalized seizures } \\
\text { Absence seizures } \\
\text { Myoclonic seizures } \\
\text { Tonic-clonic seizures }\end{array}$ & $\begin{array}{c}1(3.0 \%) \\
7(21.2 \%) \\
23(69.7 \%)\end{array}$ \\
\hline
\end{tabular}

${ }^{1}$ Multiple types possible.

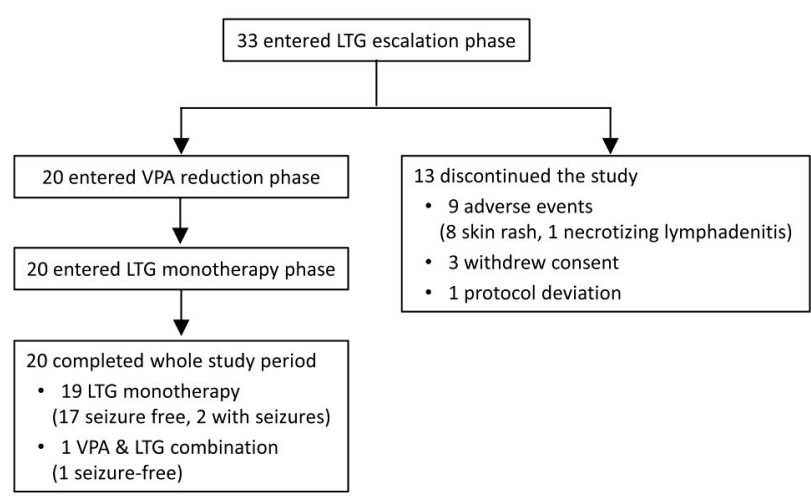

Figure 2. Patient disposition

related dizziness. The VPA dose was subsequently reduced to $0 \mathrm{mg}$ /day for the next 3-18 weeks (median: 12 weeks), and the LTG dose was increased by $52.6 \pm 24.85 \mathrm{mg} /$ day (range: $0-100 \mathrm{mg} /$ day) when the VPA dose was decreased to $0 \mathrm{mg} /$ day $(\mathrm{n}=19)$. The final LTG dose at the final visit of the LTG mono- 
therapy phase was $268.4 \pm 51.26 \mathrm{mg}$ /day (range: $150-400 \mathrm{mg} /$ day, $\mathrm{n}=19$ ). The remaining 1 patient received a VPA maintenance dose of $100 \mathrm{mg} /$ day (previous VPA dose: $600 \mathrm{mg} /$ day) and a concomitant LTG dose of $200 \mathrm{mg}$ /day based on the results of an optional electroencephalogram (EEG).

In the 20 patients, although seizures were not observed during the LTG escalation phase and VPA reduction phase at an LTG dose of 100-200 mg/day, seizures occurred once in 2 patients during the LTG monotherapy phase (one patient at 7 weeks and the other at 13 weeks after VPA removal) (Fig. 2). In both patients, tonic-clonic seizures occurred, which were the same type as their previous seizure. For these 2 patients, the LTG dose was increased from 250 to $300 \mathrm{mg} /$ day for a few days, and from 300 to $400 \mathrm{mg} /$ day for the rest of the study period. No further seizures occurred in these patients. Unless skin rash occurred, the VPA dose could be reduced and LTG monotherapy could be achieved in $95.0 \%(19 / 20)$ of the patients. For the remaining 1 patient (5.0\%), the VPA dose was reduced by more than $80 \%$. The seizure frequency was not altered for the majority of the patients $(90.0 \%)$.

\section{Safety analyses}

The most common LTG-related adverse events (reported by $\geq 5 \%$ of 33 patients) are shown in Table 2. All these adverse events have been reported previously [12, 13], and included skin rash (drug eruption or rash) in 8 patients $(24.2 \%)$, somnolence in 4 patients $(12.1 \%)$, and dizziness in 2 patients $(6.1 \%)$. The rates of somnolence and dizziness were lower than those in the prescribing information in Japan (18.5\% and 15.2\%),
Table 2. Adverse events related to LTG during the entire study period (reported by $\geq 5 \%$ of 33 patients with LTG treatment)

\begin{tabular}{cc}
\hline & $\mathrm{n}(\%)$ \\
\hline Any adverse event & $17(51.5 \%)$ \\
Drug eruption & $5(15.2 \%)$ \\
Somnolence & $4(12.1 \%)$ \\
Rash & $3(9.1 \%)$ \\
Dizziness & $2(6.1 \%)$ \\
\hline
\end{tabular}

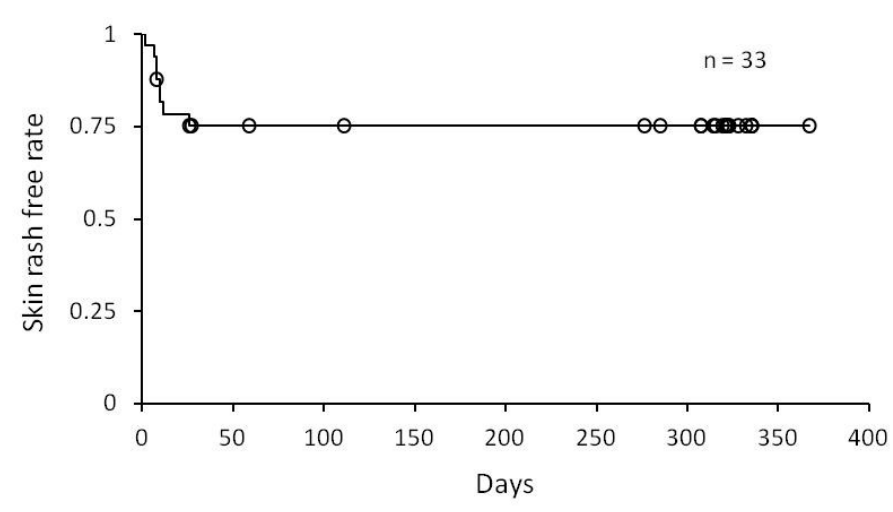

Figure 3. Kaplan-Meier plot of time to skin rash Mild to moderate skin rash occurred in 8 patients within an average of 10 days after starting LTG treatment (range: 2-26 days). The circles indicate withdrawal from the study due to non-skin rash reasons (up to 4 months) or study completion (9-12 months).

while rash occurred at a rate higher than that reported in a recent adult phase III monotherapy study conducted both in Japan and Korea (10 of 65 patients, $15.4 \%$ ) [12] and that described in the prescribing information in Japan (21 of 335 patients, 6.3\%; LTG add-on therapy). These differences in rate might be explained simply by the difference in the number of patients studied. In meta-analyses, the rate of rash was $8-11 \%$ including various conditions [13-15], and increased from $6.0 \%$ to $12.2 \%$ with concomitant use of VPA compared with LTG monotherapy [13]. As shown in Figure 3, skin rash occurred at the very 
beginning of the LTG escalation phase (within 10 days on average, range: 2-26 days) at an LTG dose of either $25 \mathrm{mg}$ every other day $(n=6)$ or $25 \mathrm{mg}$ daily $(\mathrm{n}=2)$, which was comparable to previous studies $[12,13]$.

Importantly, although skin rash occurred in some patients in this study, the impact on each individual patient was minimal. LTG treatment was discontinued within 4 days on average (range: 1-8 days) after the occurrence of any signs of LTG-related skin rash or hypersensitivity, such as fever and sore throat, which were often recognized by the patients. Hence, mild to moderate symptoms were resolved within a month on average (range: 1175 days), and did not develop into more severe conditions such as Stevens-Johnson syndrome and toxic-epidermal necrolysis.

As described in the prescribing information in Japan, since concomitant use of VPA and LTG is known to increase the risk of skin rash [13], we examined whether the higher initial VPA dose is directly related to skin rash. However, there was no significant difference between VPA doses in patients who completed the study $(\mathrm{n}=20)$ and patients who discontinued the study due to skin rash $(\mathrm{n}=8)$ (post-hoc analysis: $587.5 \pm$ 152.07 vs. $537.5 \pm 244.58 \mathrm{mg} /$ day, respectively; difference [mean $\pm \mathrm{SE}$ ]: $50.0 \pm 76.00$, 95\% CI: -106.22 to 206.22 ). Since younger age is known to be associated with a higher incidence of skin rash when using LTG [13], we examined whether the relatively younger age of our study population is a factor for the observed skin rash. However, there was no obvious trend between age and occurrence of skin rash (post-hoc analysis: $27.2 \pm 8.75$ vs. $23.8 \pm 4.92$ years for patients with and without skin rash, respectively; difference: $3.4 \pm$

\subsection{1, 95\% CI: -3.40 to 10.20$)$.}

A previous conversion study [7] showed that VPA may cause weight gain. We therefore investigated changes in weight with the reduction of VPA dose. In 20 patients with VPA dose reduction, their weights were slightly but significantly decreased from baseline at the final visit of the LTG monotherapy phase (post-hoc analysis: $56.2 \pm 9.54$ vs. $53.5 \pm 7.21 \mathrm{~kg}$; difference: $-2.70 \pm 0.745$, $95 \%$ CI: -4.255 to -1.135 ).

\section{Pharmacokinetics}

As shown in Figure 4A, during the VPA reduction phase, the LTG trough serum concentration decreased gradually along with a gradual reduction in VPA dose from 800 to $100 \mathrm{mg} /$ day, which was consistent with a previous study showing that the maximal interaction between VPA and LTG occurred at a VPA dose of $500 \mathrm{mg} /$ day and lasted until a VPA dose of $125 \mathrm{mg}$ /day was reached [16]. When VPA was removed, the addition of LTG dose of around $50 \mathrm{mg} /$ day on average caused a partial increase in its serum concentration (Fig. 4A). Although the LTG serum concentrations varied notably among the patients (Fig. 4B), the LTG concentration at the final visit of the LTG monotherapy phase was $7.2 \pm 3.56 \mathrm{mg} / \mathrm{mL}$ at an LTG dose of $150-400$ $\mathrm{mg}$ /day $(\mathrm{n}=19)$, and the LTG concentration decreased apparently from the first visit of the VPA reduction phase with an LTG dose of 100-200 mg/day. In 1 patient, the LTG serum concentration decreased to $0.63 \mathrm{mg} / \mathrm{mL}$ when seizure occurred during the LTG monotherapy phase, which was due to noncompliance, but returned to $4.81 \mathrm{mg} / \mathrm{mL}$ at the last visit of the same phase. 

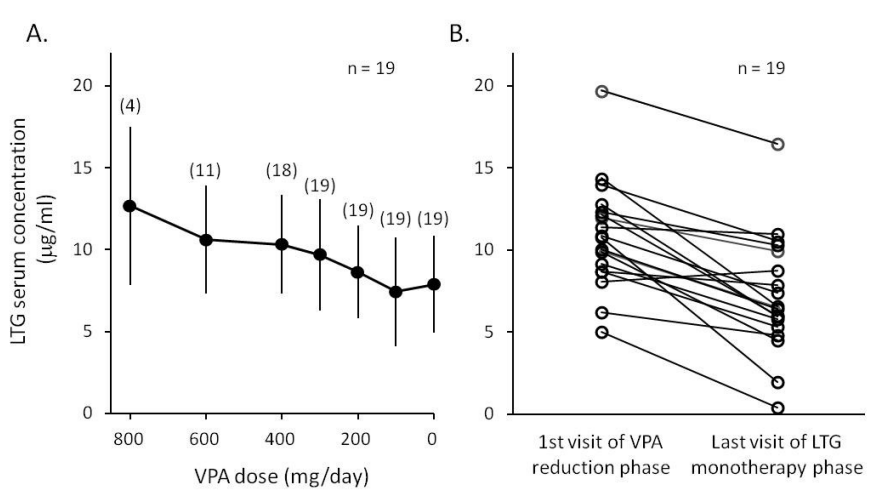

Figure 4. LTG pharmacokinetics

A: Mean LTG trough serum concentrations at different VPA doses in 19 patients who achieved LTG monotherapy. Data with more than 4 patients are shown. The number in parentheses represents number of patients included in the analysis. Error bars indicate standard deviation. B: Raw data of 19 patients at the first visit of the VPA reduction phase and at the last visit of the LTG monotherapy phase, respectively.

\section{Change in QOL scores}

In the 20 patients who completed the entire study period, the changes in various aspects of QOL were assessed. The scores in QOLIE31-P or QOLIE-AD-48 did not change notably (Fig. 5A, B) between baseline and the last visit of the LTG monotherapy phase.
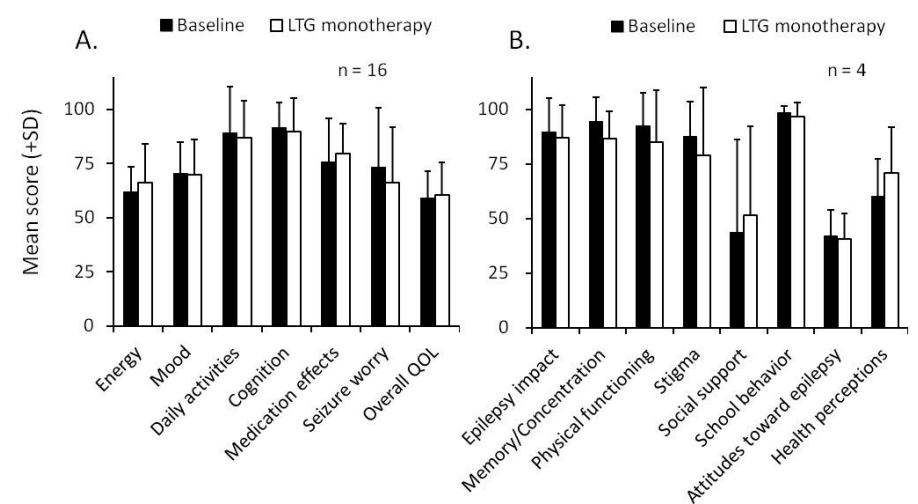

Figure 5. Scores of QOL.

The QOLIE-31-P [10] and QOLIE-AD-48 [11] were used for patients aged $\geq 18$ (A) and $<18$ (B), respectively. Only 20 patients who completed the entire study period were included. Black and white bars indicate scores of various aspects at baseline (week 0) and at the last visit of the LTG monotherapy phase, respectively. Error bars indicate standard deviation.

\section{Discussion}

This study showed that by paying careful attention to the occurrence of skin rash or hypersensitivity at the very beginning of the LTG escalation phase, it was possible to change VPA monotherapy to LTG monotherapy with 10-17 weeks of LTG escalation and subsequently 3-18 weeks of VPA reduction, without substantially altering seizure frequency. To minimize the risk of break-through seizures, the VPA dose was reduced after the LTG dose had reached $200 \mathrm{mg}$ /day. However, in clinical practice, it may be plausible to start reducing the VPA dose while increasing the LTG dose, once the added LTG dose shows efficacy comparable to that of the initial VPA dose.

A conversion algorithm similar but faster than the one used in the present study was used in a study of topiramate (TPM), another new AED, in which TPM was added onto VPA monotherapy at $25-\mathrm{mg}$ /day increment once every 1-2 weeks [17]. Upon reaching a TPM dose of 50-200 $\mathrm{mg}$ /day, the VPA dose was decreased to $0 \mathrm{mg} /$ day. Thus, TPM monotherapy was achieved within 7 weeks on average. However, the risk of malformation when using TPM is more than twice as high as LTG [2], and TPM monotherapy has not been approved in Japan. There is no relevant clinical trial on switching to other new AEDs including levetiracetam.

Skin rash occurred even when the approved dosing regimen of LTG was strictly followed, although the development of skin rash is usually associated with a faster LTG escalation regime [13]. Previous metaanalyses have reported several risk factors for skin rash [12-15], but we did not find any 
such risk factors probably because of the small sample size of our study. Rather, the relatively high incidence of skin rash observed in this study may be explained by the nature of clinical trials that are conducted under strict procedures.

It is important to provide clear instructions to patients and encourage them to visit their doctors upon noticing any initial sign of skin rash. These initial signs include fever as is also found in common cold and influenza, as well as headache and nausea. Once skin rash occurs, LTG should be discontinued immediately unless the rash is considered unrelated to the use of LTG. Consultation with a dermatologist may help to obtain a definitive diagnosis and ensure that the symptoms resolve.

Since seizures occurred in 2 patients during the LTG monotherapy phase (one seizure episode in each patient), an LTG dose of 200 $\mathrm{mg} /$ day may have been inadequate to control seizures. Therefore, further adjustment of LTG dose would be necessary. In the study by Sale et al. [6], in which the approved dosing regimen was different from that in Japan, the LTG dose was increased by $100 \mathrm{mg}$ /day at the last 2 steps (200 to $400 \mathrm{mg} /$ day) before removal of the VPA dose, and was maintained at an LTG dose of $500 \mathrm{mg}$ /day during the LTG monotherapy phase. Moreover, in some cases, the timing of VPA dose reduction was determined by a trend of decrease in LTG trough serum concentration. Thus, measurement of LTG serum concentration may be useful in minimizing the risk of seizures.

The patients' QOL scores did not improve, probably because the patients enrolled were seizure-free even when they were on VPA monotherapy. Rather, the result seems to indicate that the conversion from VPA monotherapy to LTG monotherapy can be accomplished without affecting the patients' QOL notably.

This study proposed a practical methodology to help physicians change treatment of patients in their clinic. The risk of fetal malformation [2] and cognitive developmental deficits [3] may be reduced by reducing VPA dose. Therefore, the conversion may be beneficial in women of child bearing potential who wish to become pregnant. A recent report provides further guidance on the use of VPA in various clinical situations based on risk-benefit assessments of different treatment options [18].

\section{Acknowledgements}

We thank all the investigators of the Lamictal 200776 Study Group who participated in the study (see below). This study was sponsored by GlaxoSmithKline K.K. (study number: 200776), and all data management and analyses were conducted by the Biomedical Data Sciences Department of GlaxoSmithKline K.K. The pharmacokinetic analyses and medical monitoring were conducted by SRL Co., Ltd. and A2 Healthcare Corporation, respectively, under contract with GlaxoSmithKline K.K. HM, HO, and YN are full-time employees of GlaxoSmithKline K.K., and HM and YN are stockholders of GlaxoSmithKline. TY has received lecture fees from GlaxoSmithKline K.K. and Otsuka Pharmaceutical Co., Ltd. YK has received lecture fees from GlaxoSmithKline K.K. AI has received lecture fees from Otsuka Pharmaceutical Co., Ltd., GlaxoSmithKline K.K., 
and UCB Japan Co., Ltd. The Department of Epilepsy, Movement Disorders and Physiology at Kyoto University Graduate School of Medicine is an endowment department supported by grants from GlaxoSmithKline K.K., Nihon Kohden Corporation, Otsuka Pharmaceutical Co., Ltd., and UCB Japan Co., Ltd.

\section{The Lamictal 200776 Study Group:}

Drs. S. Ito, Y. Kubota, H. Nakamoto, N. Oshiro (Asakadai Central General Hospital), M. Kato, T. Onuma, M. Sekimoto (Musashino-Kokubunji Clinic), H. Enoki, A. Fujimoto, T. Miyagi, T. Okanishi, T. Yamamoto, T. Yamazoe, T. Yokota (Seirei Hamamatsu General Hospital), A. Ikeda, T. Inoue, T. Kato, K. Kobayashi, R. Matsumoto, N. Sawamoto, A. Shimotake, R. Takahashi (Kyoto University), M. Moriya, M. Nakano (Toyonaka Municipal Hospital), N. Kamio, K. Miki, H. Ninomiya (Itami Municipal Hospital), and S. Tanaka (Tanaka Neurosurgical Clinic).

\section{References}

[1] Japanese Society of Neurology: Guidelines for the treatment of epilepsy 2010. Available at http://www.neurologyjp.org/guidelinem/tenkan.html.

[2] Hernandez-Diaz S, Smith CR, Shen A, Mittendorf R, Hauser WA, Yerby M, Holmes LB. Comparative safety of antiepileptic drugs during pregnancy. Neurology 2012; 78: 1692-9.

[3] Meador KJ, Baker GA, Browning N, Cohen MJ, Bromley RL, Clayton-Smith J, Kalayjian LA, Kanner A, Liporace JD, Pennell PB, Privitera M, Loring DW.
Fetal antiepileptic drug exposure and cognitive outcomes at age 6 years (NEAD study): a prospective observational study. Lancet Neurol 2013; 12: 244-52.

[4] FDA Drug Safety Communication: Valproate anti-seizure products contraindicated for migraine prevention in pregnant women due to decreased IQ scores in exposed children (05-06-2013). Available at http://www.fda.gov/Drugs/ DrugSafety/ucm350684.htm.

[5] Jozwiak S, Terczynski A. Open study evaluating lamotrigine efficacy and safety in add-on treatment and consecutive monotherapy in patients with carbamazepine- or valproateresistant epilepsy. Seizure 2000; 9: 48692.

[6] Sale ME, Natarajan S, Biton V, Vuong A, Hammer AE, Messenheimer JA, Blum D. A dosing algorithm for converting from valproate monotherapy to lamotrigine monotherapy in patients with epilepsy. Epilepsy Behav 2005; 6: 63-70.

[7] Buchanan N. The use of lamotrigine in juvenile myoclonic epilepsy. Seizure 1996; 5: 149-51.

[8] Morris GL, Hammer AE, Kustra RP, Messenheimer JA. Lamotrigine for patients with juvenile myoclonic epilepsy following prior treatment with valproate: results of an open-label study. Epilepsy Behav 2004; 5: 509-12.

[9] Yuen AW, Land G, Weatherley BC, Peck AW. Sodium valproate acutely inhibits lamotrigine metabolism. $\mathrm{Br} \mathrm{J}$ Clin Pharmacol 1992; 33: 511-3.

[10]Cramer JA, Van Hammee G. Maintenance of improvement in health- 
related quality of life during long-term treatment with levetiracetam. Epilepsy Behav 2003; 4: 118-23.

[11] Cramer JA, Westbrook LE, Devinsky O, Perrine K, Glassman MB, Camfield C. Development of the Quality of Life in Epilepsy Inventory for Adolescents: the QOLIE-AD-48. Epilepsia 1999; 40: 1114 -21 .

[12] Yamamoto T, Hong SB, Shimizu M, Sato K, Numachi Y. Lamotrigine monotherapy in newly diagnosed epilepsy or recurrent epilepsy: A multicenter, open-label study. Epilepsy \& Seizure 2014; 7: 55-65.

[13] Messenheimer J, Mullens EL, Giorgi L, Young F. Safety review of adult clinical trial experience with lamotrigine. Drug Saf 1998; 18: 281-96.

[14]Alvestad S, Lydersen S, Brodtkorb E. Rash from antiepileptic drugs: influence by gender, age, and learning disability. Epilepsia 2007; 48: 1360-5.

[15] Wang XQ, Lang SY, Shi XB, Tian HJ, Wang RF, Yang F. Antiepileptic druginduced skin reactions: a retrospective study and analysis in 3793 Chinese patients with epilepsy. Clin Neurol Neurosurg 2012; 114: 862-5.

[16]Gidal BE, Sheth R, Parnell J, Maloney $\mathrm{K}$, Sale M. Evaluation of VPA dose and concentration effects on lamotrigine pharmacokinetics: implications for conversion to lamotrigine monotherapy. Epilepsy Res 2003; 57: 85-93.

[17] Schreiner A, Stollhoff K, Ossig W, Unkelbach S, Luer W, Bogdanow M, Schauble B. Conversion from valproic acid onto topiramate in adolescents and adults with epilepsy. Acta Neurol Scand
2009; 119: 304-12.

[18] Tomson T, Marson A, Boon P, Canevini MP, Covanis A, Gaily E, Kalviainen R, Trinka E. Valproate in the treatment of epilepsy in girls and women of childbearing potential. Epilepsia 2015;

56: 1006-19. 